\title{
How creation of a parent satisfaction questionnaire improved multidisciplinary service delivery in a paediatric day surgery unit.
}

\author{
Alison Bradley
}

\begin{abstract}
Auditing patient satisfaction has become a keystone of quality patient centred healthcare. A plethora of patient satisfaction studies exist but only a few studies have been evaluated for their validity, reliability, specificity or psychometric properties. And the majority focus on adult health care. However, if validated tools are not utilised, then inaccurate results could stymie service improvement.
\end{abstract}

The level of satisfaction with the paediatric day surgery service at Tayside Children's Hospital was unknown. Our objective was to measure parent satisfaction with the paediatric day surgery by creating a parent satisfaction questionnaire which has undergone satisfactory testing for validity, reliability, specificity and psychometric properties. A Likert-style questionnaire was constructed through literature review and focus group meetings with professionals, parents and patient groups to establish content validity. Statements worded in positive phrasing were reworded in negative phrasing to ensure intra-rater reliability. A pilot study was conducted and responses analysed for construct validity and inter-rater agreement. Internal reliability was established using Chronbach's alpha analysis, which produced scores for each part of the questionnaire between 0.7 and 0.9 .

Overall parent satisfaction was high. $95.48 \%$ either strongly agreed or agreed with positively worded statements regarding pre-operative clinic service. In particular $100 \%$ satisfaction was reported with the pre-operative phone call which only $70 \%$ of participants received. $96.60 \%$ strongly agreed or agreed with positive statements regarding service provided on the ward and $87.50 \%$ strongly agreed or agreed with positive statements regarding the discharge process. $5 \%$ specifically requested improved information giving.

In conclusion the parent satisfaction questionnaire was found to have proven validity, reliability, specificity and psychometric properties. Overall parent satisfaction was found to be high. Areas identified for improvement included delivering pre-operative phone call to all parents who have children undergoing day surgery and further exploration of sources and methods of information giving.

\section{Problem}

The level of parent satisfaction with the overall service of the paediatric day surgery in Tayside Children's Hospital was unknown. Furthermore, there is a lack of existing validated tools available to measure parent satisfaction with paediatric day surgery. The challenge of addressing this problem was compounded by the fact that several multidisciplinary teams, attached to different surgical specialities, were operating within a small paediatric day case unit on different days of the week. Some of these teams had professionals who specialised only in paediatric surgery whilst others dealt mainly with adults and occasionally operate a paediatric list. Therefore the pre-operative referral system varied significantly between patients. Furthermore, staff from the various pre and post-operative clinics were rarely afforded the opportunity to meet with health professionals working either on the surgical unit or in other clinics.

Previous attempts to measure patient/ parent satisfaction were led by health professionals often working either in isolation or only within their own discipline. The questions put forward to the parents were therefore heavily biased by the professional paradigm of the questionnaire's author. However numerous studies show that patient perception of quality of health care differs from that of professionals [3].

As a consequence of auditing parent satisfaction without a deeper understanding of the concept of patient satisfaction, very little was known about levels of parent satisfaction with the overall service of the paediatric day case surgery.

\section{Background}

Patient satisfaction has gained precedence within healthcare [1-3]. Numerous studies show that patient perception of quality of health care differs from that of professionals [3]. Auditing patient satisfaction has therefore become a keystone of quality patient centred healthcare delivery [1-3]. This means improved employee support and training is required, which should also enhance employee satisfaction [1]. However, if validated tools are not utilised, then inaccurate results may stymie service improvement.

A plethora of patient satisfaction studies exist. However, the majority focus on adult healthcare and few studies had used questionnaires which had been evaluated for their validity, reliability, specificity or psychometric properties [3]. Furthermore 
questionnaires should be developed or adapted for specific healthcare settings to allow reliable comparisons. Both day case and paediatric settings differ significantly from other inpatient settings and so do the needs and expectations of paediatric patients and their parents.

A literature search using Cochrane Library, Medline, PubMed, EMBASE, OvidSP, CINAHL, and googlescholar databases aimed to evaluate instruments thus far devised to measure parent satisfaction with paediatric day surgery. Search terms included: patient satisfaction, parent satisfaction, paediatric patient satisfaction, paediatric surgery, paediatric day surgery and day surgery. This search revealed that there is a lack of studies within the past 10 years exploring parent satisfaction with paediatric health care. Specifically there is a dearth of studies looking at parent satisfaction with paediatric day surgery.

Only one study within the past 10 years by Bittmann \& Ulus focused on parent satisfaction with day surgery [4]. This study included 136 parents whose children underwent: urological, plastics and emergency day-case operations performed by two paediatric surgeons using the same anaesthetic team. Although Chronbach's alpha coefficient was used to prove reliability, no further tests of questionnaire validity were undertaken. Furthermore the three model criteria of satisfaction with: surgical staff /general treatment, general information giving and aesthetic staff were decided by the authors alone based on Kaiser Criterion. The questionnaire focused on identifying post-operative symptoms including nausea, vomiting and pain. Therefore potential aspects of satisfaction, deemed important by parents, may not have been measured. Another study focused on parent satisfaction with ENT day surgery but fell out of the 10 year period and was limited by lack of validity, reliability or psychometric testing of the questionnaire [5]. Two studies used questionnaires adapted from previously validated questionnaires but comparability was limited as they focused solely on satisfaction with anaesthetic care and patients with cerebral palsy respectively $[1,6]$.

In summary there is a lack of up-to-date quality research exploring parent satisfaction with paediatric day surgery.

\section{Baseline measurement}

The aims of this project were:

1) To produce a parent satisfaction questionnaire which is suitable for use in paediatric day case units. The questionnaire must undergo satisfactory testing for validity, reliability, specificity and psychometric properties.

2) Produce a questionnaire which allows underlying dimensions of parent satisfaction to be derived and individual scores calculated.

3)To illicit aspects of health care delivery within the paediatric day surgery service that could be improved.

\section{Design}

A Likert-style questionnaire was constructed around the existing structure of the paediatric day surgery service to quantify the dimensions of parent satisfaction. Section A collected demographic information. Section $B$ assessed satisfaction with pre-operative clinic services. Section $C$ assessed service provision on the surgical ward and Section $D$ assessed satisfaction with discharge procedures.

The questionnaire was constructed in a stepwise process to maximise validity and reliability $[2,3,7]$. Opinions regarding positive and negative aspects of the service were gathered from: surgeons, anaesthetists, charge nurses, staff nurses, theatre nurses, representatives from parental patient groups, health care assistants, play therapists, doctors, medical and nursing students. The opinions were gathered through face-to-face interviews, emails, and anonymous suggestion boxes placed on the ward and in clinics. This, in addition to a literature review, created a pool of items for inclusion in the questionnaire.

A pilot study was conducted and responses tested for validity and reliability. Literature review and focus group meetings established content validity $[2,8]$. Face validity, a subjective assessment by the author of whether the questionnaire measures outcomes of parent satisfaction with paediatric day surgery, was therefore also accepted $[2,8]$. Construct validity was established by demonstrating that parents who strongly agreed or agreed with positive statements disagreed of strongly disagreed with negative statements about the same aspect of their child's care and vice versa $[2,8]$. Criterion validity is the correlation of a measure with an accepted goldstandard criterion measure [2,8]. The latter does not yet exist for parent satisfaction with paediatric day surgery and therefore could not be established. Statements worded in positive phrasing were reworded in negative phrasing to ensure intra-rater reliability. Inter rater agreement was also demonstrared. Internal reliability was established with Chronbach's alpha scores between 0.7 and 0.9 for each section of the questionnaire.

Parents/guardians of children receiving elective day surgery were invited by nursing staff to complete the anonymous questionnaire at the time of discharge, after the discharge information had been given. Questionnaires were administered over an 8 month period. Results were disseminated through departmental and national meetings and international conference.

\section{Strategy}

\section{PDSA Cycle 1}

Senior medical and nursing staff were approached to discuss what was already known about levels of parent satisfaction with the paediatric day surgery service and to analyse previous attempts at measuring parent satisfaction. As predicted, little was known about levels of parent satisfaction with the overall service as previous attempts at measuring satisfaction were sporadic and tended to focus on either clinic or ward settings and lacked a multidisciplinary approach and input from parents. As a result, it was agreed to look at the service as a whole from pre-operative clinics to discharge and measure levels of parent satisfaction. 
PDSA Cycle 2

A literature review was conducted to analyse existing instruments to evaluate parent/guardian satisfaction. This showed that there was a lack of existing tools with proven validity and reliability that were suitable for use when measuring parent satisfaction with paediatric day surgery. This provided a useful guide for constructing a questionnaire with proven validity and reliability. After consultation with senior nursing and medical staff it was decided to create our own parent satisfaction questionnaire.

PDSA Cycle 3

Focus groups were set up with all multidisciplinary professionals as well as parent patient groups to discuss positive and negative aspects of the service and to establish dimensions of satisfaction. As predicted, views on what constituted parent satisfaction differed slightly between parents and professionals. Responses were compiled into a pool of items for inclusion in the questionnaire.

\section{PDSA Cycle 4}

The appropriateness and comprehensiveness of the pool of items for inclusion in the questionnaire was discussed and agreed with all those consulted in PDSA Cycle 3. Some professionals initially wanted to exclude items they deemed as being less important with regards to how responses would help them to improve their own practice. These items were however included owing to their perceived importance to parents and other members of the multidisciplinary team. A draft version of the questionnaire was therefore constructed by refining the pool of items into a questionnaire with a Likert-style scale giving responses ranging from strongly agree to strongly disagree.

\section{PDSA Cycle 5}

The questionnaire was refined further following discussions and qualitative feedback from those consulted in PDSA Cycle 3 Balancing comprehensiveness of the questionnaire whilst keeping it to an appropriate length was a challenge. The inclusion of a free text box for additional comments was agreed. Some professionals suggested not rewording questions in a positive phrasing also in a negative phrasing to reduce the length of the questionnaire. However as this would ensure intra-rater reliability it was agreed that this should stay in unless the pilot study provided evidence that the questionnaire was too long in which case a further review was planned $[2,8]$.

\section{PDSA Cycle 6}

A pilot study was conducted including a free text box inviting parents to give qualitative feedback on the questionnaire. 28 out of 50 questionnaires were completed and returned for the pilot study. All questionnaires were completed in full and participants either did not comment or commented positively on the questionnaire. Results were analysed and validity and reliability of the questionnaire was proven.

\section{PDSA Cycle 7}

A final version of questionnaire was produced and administered by nursing staff to parents of children undergoing elective paediatric day surgery in Tayside Children's Hospital after all discharge information and paperwork had been completed. The project ran over an 8 month period.

\section{Results}

Of the 150 questionnaires made available 94 were returned. 8 were excluded as they were incomplete with only minimal demographic information given.

Overall parent satisfaction was high. $95.48 \%$ either strongly agreed or agreed with positively worded statements regarding preoperative clinic service. $96.60 \%$ strongly agreed or agreed with positive statements regarding service provided on the ward and $87.50 \%$ strongly agreed or agreed with positive statements regarding the discharge process.

In particular, $100 \%$ of those who received a phone call prior to admission either strongly agreed or agreed with positive statements regarding this aspect of the service. This phone call included further information giving about fasting, admission procedure and gave parents the opportunity to ask any further questions. Its popularity with parents had not previously been realised and only $70 \%$ of parents completing the questionnaires received this service. An audit is consequently underway to improve delivery of this service to all parents whose children are undergoing elective day surgery.

A small number (5\%) requested more information be given pre and post-operatively. However $14 \%$ did not record either consultant or type of operation despite completing the rest of the questionnaire correctly. A significant number only gave the vague anatomical location operated on. This would infer a need for improved information giving and mandate a further study exploring satisfaction with sources and mechanisms of information giving.

\section{Lessons and limitations}

Although there are few studies to compare these results to, it is interesting to note that the questionnaire centred on similar domains (communication/information giving, environment and patient care) to those devised through similar processes for different paediatric hospital settings $[1,3,4,6-9]$. Also, by consulting the entire multidisciplinary team as well as parents, some items included in this questionnaire were not included in previous parent satisfaction questionnaires designed by professionals alone. The popularity of the pre-operative phone call service had therefore been overlooked.

This project was limited by small sample size. It is hoped that by running this questionnaire at multiple paediatric day surgery units across Scotland we can increase sample size and learn from positive and negative aspects of service across different settings. By repeating the questionnaire at a fixed time post discharge, testretest reliability of the questionnaire could be established and would remove potential bias since staff knew the study was in progress and parents may have felt obliged to respond positively because they were still on the ward when completing the questionnaire. Using this project as a basis, further research is mandated in areas of sources/ methods of information giving and challenges of 
including illiterate and non-English speaking parents in future studies.

\section{Conclusion}

The parent satisfaction questionnaire was found to have proven validity, reliability, specificity and psychometric properties. This enabled accurate measurement of parent satisfaction.

Overall levels of parent satisfaction with the paediatric day surgery in Tayside Children's Hospital are high. Patient care, however, has still managed to improve. This project highlighted that $100 \%$ of participants who received a phone call prior to admission either strongly agreed or agreed with positive statements regarding this aspect of the service, yet only $70 \%$ were receiving such a phone call. A further audit is therefore underway to improve delivery of this service. A small number requested more information be given pre and post-operatively.

However $14 \%$ did not record either consultant or type of operation despite completing the rest of the questionnaire correctly. A significant number only gave the vague anatomical location of their child's operation. Further research, using this tool as a basis, is mandated in areas of sources and methods of information giving and challenges of including illiterate and non-English speaking parents in future studies.

\section{References}

1. Lew VK, Lalwani K, Palermo TM. Factors affecting parental satisfaction following pediatric procedural sedation. Journal of Clinical Anesthesia. 2010;22:29-34

2. Chanthong P, Abrishami A, Wong J, Herrera F, Chung F. Systematic review of questionnaires measuring patient satisfaction in ambulatory anesthesia. Anesthesiology 2009;110:1061-1067

3. Gonzalez N, Quintana JM, Bilbao A, Escobar A, Aizpuru F, Thompson A, Esteban C, Antonio J, Sebastian JAS, La Sierra ED. Development and validation of an in-patient satisfaction questionnaire. Int Journal for Quality in Health Care. 2005;17:6:456-472

4.Bittmann S, Ulus H. Parent satisfaction with paediatric daysurgery: a questionnaire-based study. J of Ambulatory Surgery. 2004;11:3-5

5.Hicklin L, Tostevin PMJ, Wyatt ME. Parental satisfaction with paediatric day0case ENT surgery. The Journal of Laryngology and Otology.1999;113:1072-1075

6. Lee SH, Ching CY, Park MS, Choi H, Cho TJ, Yoo WJ, Lee KM. Parental satisfaction after single-event multilevel surgery in ambulatory children with cerebral palsy. J Pediatric Orthopaedics. 2009;29:4:398-401

7. McPherson ML, Sachdeva RC, Jefferson LS. Development of a survey to measure parent satisfaction in a pediatric intensive care unit. Crit Care Med.2000;28:8:3009-3013

8 .Ygge BM, Arnetx JE. Quality of paediatric care: application and validation of an instrument for measuring parent satisfaction with hospital care. Int J for Quality in Health Care. 2001;13:1:33-43

9. Welch SJ. Twenty years of patient satisfaction research applied to the emergency department: a qualitative review. American Journal of Medical Quality. 2010;25:64-72

\section{Declaration of interests}

Nothing to declare.

\section{Acknowledgements}

Amanda McCabe, Rodney Grant, Suzanne Byres, all the staff working in Tayside Children's Hospital paediatric surgical unit. 